\title{
Enhancing the Efficiency and Accuracy of MLFMA-PO Hybrid Method for Analyzing Electrically Large Objects
}

\author{
Hao Ding, Yong Zhang, and Hai Lin \\ State Key Laboratory of CAD\&CG, Zhejiang University, Hangzhou 310058, China \\ Correspondence should be addressed to Hai Lin; lin@cad.zju.edu.cn
}

Received 18 December 2014; Revised 9 March 2015; Accepted 14 March 2015

Academic Editor: Miguel Ferrando Bataller

Copyright ( 2015 Hao Ding et al. This is an open access article distributed under the Creative Commons Attribution License, which permits unrestricted use, distribution, and reproduction in any medium, provided the original work is properly cited.

\begin{abstract}
An enhanced hybrid technique combining the multilevel fast multipole algorithm (MLFMA) and the physical optics (PO) is presented for analyzing scattering and radiation by electrically large structures. Based on the previously proposed hybrid technique, an extra octree structure is built for the moment method (MoM) region to accelerate the solving process and an iteration scheme is proposed to further enhance the efficiency compared to the conventional hybrid method. What is more, the modified iterative physical optics (IPO) accelerated by MLFMA technique is adopted to improve the accuracy by simulating complex structures with strong reflections. Numerical results are given to demonstrate the improved accuracy and higher accuracy of the proposed hybrid technique compared to the previous MLFMA-PO method.
\end{abstract}

\section{Introduction}

Nowadays, simulations of large-scale electromagnetic problems using hybrid techniques which combine the accurate numerical methods and asymptotic high-frequency methods have achieved more and more attention in the area of computational electromagnetics (CEM). Among them, the hybrid technique which combines the integral numerical method based on the conventional MoM [1] and the high-frequency method based on physical optics (PO) is very popular and efficient. There are usually two different ways to implement the MoM-PO: (1) considering the impedance matrix of the MoM by coupling the $\mathrm{PO}$ contributions [2,3]; (2) iterating the voltage matrix of the MoM by PO currents [4-6]. The former one is self-consistent while the iterative MoM-PO is more efficient without losing any accuracy. Therefore, the iterative MoM-PO is widely studied and used in the CEM area.

Since the accuracy of conventional MoM-PO solution is limited, one apparent idea is to enlarge the area of the MoM region at the cost of losing efficiency. More unknowns in MoM region will not only cause a heavy burden for the local solver but also increase the complexity of the coupling between the different regions. Several algorithms have been proposed to alleviate this problem such as high-order hybrid
MoM-PO techniques [7], multilevel fast multipole algorithm (MLFMA) [8], precorrected-FFT (P-FFT) [9], and adaptive integral method (AIM) [10]. Among them, the MLFMA-PO hybrid technique $[11,12]$ seems to be the most efficient one since the MLFMA is proved to be the most efficient fast algorithm for 3D arbitrary objects. In our previous work, MLFMA is adopted to accelerate not only the solution of the matrix equations in the MoM region, but also the interactions between the MoM region and PO region. By using this scheme, the computational complexity can be reduced to less than $O(N \log (N))$.

Many correction techniques have been proposed to improve the hybrid MoM-PO method since the accuracy of PO method is not sufficiently enough for the analysis of complex structures. Among them, two main methods are introduced here. The first approach is to add certain correction terms on the wedges [13-15] which take the influences of sharp edges into consideration. The other approach is the iterative PO method $[16,17]$ which considers the mutual influences in the PO region. The second approach is simple and suitable for structures with strong reflections such as concavities.

In this paper, we are aiming at enhancing the efficiency and accuracy of the previous MLFMA-PO technique for 
simulating the radiation and scattering problems in terms of electrically large structures based on [12]. Since the burden of the matrix-vector-product (MVP) based on the octree for the whole structure is heavy and unnecessary for the MoM region, an extra octree is built for the MoM region to decrease the calculation time of the MVP in the MoM region. Based on the additional octree, an iterative scheme is proposed to further enhance the efficiency and the detailed explanation is given. Moreover, due to the limited accuracy of PO when facing the complex structures with strong reflections, the IPO is developed to improve the accuracy by considering the interactions in the high-frequency domain inspired from $[16,17]$. Several numerical results are given to demonstrate that the proposed method equipped with these corrections is more accurate and efficient compared to the conventional MLFMA-PO technique.

\section{Formulations}

Firstly, a brief review on the hybrid MoM-PO is given for completeness. As shown in Figure 1, the structure is first divided into two parts as follows: the MoM region and $\mathrm{PO}$ region, where $\mathbf{J}_{\mathrm{MoM}}$ and $\mathbf{J}_{\mathrm{PO}}$ denote the electric currents in the MoM and PO region, respectively. The coupled electric field integral equation (C-EFIE) for the MoM region $\left(S_{\mathrm{MoM}}\right)$ can be built as [2]

$$
\widehat{n} \times\left(\mathbf{L}^{E}\left(\mathbf{J}_{\mathrm{MoM}}\right)+\mathbf{L}^{E}\left(\mathbf{J}_{\mathrm{PO}}\right)\right)=\widehat{n} \times \mathbf{E}^{\mathrm{inc}},
$$

where $\widehat{n}$ denotes the unit vector in the MoM region surface $S_{\mathrm{MoM}}$ and $\mathbf{E}^{\text {inc }}$ stands for the electric field of the incident plane wave if it exists. The integral operator $\mathbf{L}^{E}$ is defined as

$$
\mathbf{L}^{E}(J)=-j k Z \int\left[\mathbf{J}+\frac{1}{k^{2}} \nabla\left(\nabla^{\prime} \cdot \mathbf{J}\right)\right] G d \mathbf{r}^{\prime},
$$

where $k$ represents the free space wave number and $Z=$ $\sqrt{\mu_{0} / \varepsilon_{0}}$ is wave impedance. $G\left(\mathbf{r}, \mathbf{r}^{\prime}\right)=e^{-j k\left|\mathbf{r}-\mathbf{r}^{\prime}\right|} /\left(4 \pi\left|\mathbf{r}-\mathbf{r}^{\prime}\right|\right)$ is free space Green's function. The electric currents in PO region which are induced by the MoM currents and incident wave (if it exists) can be written as

$$
\mathbf{J}_{\mathrm{PO}}=2 \delta \widehat{n} \times\left(\mathbf{L}^{H}\left(\mathbf{J}_{\mathrm{MoM}}\right)+\mathbf{H}^{\mathrm{inc}}\right),
$$

where $\delta$ is the shadowing effect coefficient, which is 1 in the lit PO region and 0 in the shadowed PO region. $\mathbf{H}^{\text {inc }}$ denotes the magnetic field of the incident wave. The integral operator $\mathbf{L}^{H}$ is defined as

$$
\mathbf{L}^{H}(J)=-\int \mathbf{J} \times \nabla G d \tau^{\prime} .
$$

We use the Rao-Wilton-Glisson (RWG) basis function [18] and Galerkin's testing procedure to derive the matrix equation which is shown as

$$
\begin{gathered}
\left(\mathbf{Z}_{\mathrm{MoM}}+\mathbf{A}_{\mathrm{MoM}-\mathrm{PO}} \mathbf{B}_{\mathrm{PO}-\mathrm{MoM}}\right) \mathbf{I}_{\mathrm{MoM}} \\
=\mathbf{V}_{\mathrm{MoM}}-\mathbf{A}_{\mathrm{MoM}-\mathrm{PO}} \mathbf{I}_{\mathrm{PO}},
\end{gathered}
$$

where $\mathbf{I}_{\mathrm{MoM}}$ is the current weights in the MoM region, $\mathbf{Z}_{\mathrm{MoM}}$ stands for the impedance matrix in the MoM region,

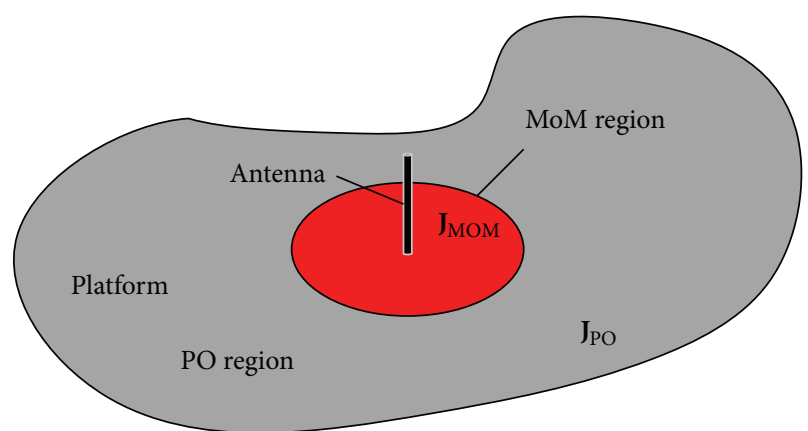

FIGURE 1: The structure is divided into MoM region and PO region.

$\mathbf{A}_{\mathrm{MoM}-\mathrm{PO}}$ and $\mathbf{B}_{\mathrm{PO}-\mathrm{MoM}}$ represent the coupling matrix between the MoM region and the PO region, and the $\mathbf{V}_{\mathrm{MoM}}$ denotes the excitations for the MoM region. $\mathbf{I}_{\mathrm{PO}}$ stands for the currents in $\mathrm{PO}$ region induced by the incident plane wave if it exists. The element expressions of matrices $\mathbf{Z}_{\mathrm{MoM}}$ and $\mathbf{A}_{\mathrm{MoM}-\mathrm{PO}}$ are the same as the conventional MoM equations with RWG basis functions. However, the element expression of matrix $\mathbf{B}_{\mathrm{PO}-\mathrm{MoM}}$ is a little different which is written as

$$
\mathbf{B}_{\mathrm{PO}-\mathrm{MoM}, i j}=\delta\left(\widehat{t}_{i}^{+}+\widehat{t}_{i}^{-}\right) \cdot \widehat{n}_{i} \times \nabla \int_{T_{j}^{ \pm}} G\left(r, r^{\prime}\right) \mathbf{f}_{j}\left(r^{\prime}\right) d \tau^{\prime},
$$

where $\hat{t}_{i}^{+}$and $\hat{t}_{i}^{-}$are the two unit vectors in the middle of the $i$ th edge which are perpendicular to the edge lying in the plane of the triangular pairs $T_{i}^{ \pm}$in the PO region. $T_{j}^{ \pm}$is the triangular pairs of the $j$ th RWG basis function.

2.1. Extra Octree Structure. In the previous implementation of MLFMA-PO hybrid technique [12], an octree structure is built based on the whole geometry information. The CEFIE is constructed on the MoM region and the impedance matrix consists of influences from itself and the PO region which are described by $Z, A$, and $B . Z$ describes the selfinteractions in the MoM region and the currents in the PO region are not needed if the MVP process is performed on $Z$. In fact, all the MVP processes are accelerated by MLFMA based on the total octree in the previous hybrid technique which is time-consuming and unnecessary. What is more, the number of unknowns in the MoM region is always much smaller than the one in PO region, and the scale of the octree built for the MoM region will be much smaller compared to the whole one. Thus, higher efficiency can be achieved in the self-interactive MVP compared to the previous MLFMA-PO hybrid technique. Even though a little more memory usage is needed for the extra tree structures, transfer matrix, interpolation matrix, and the radiation and receiving patterns in the lowest level, it is negligible compared to the whole octree structure. It should be noted that the near-field matrix for the MoM region is also different from the conventional MLFMA$\mathrm{PO}$ technique because of the different near-tree judgements.

2.2. Iteration/Noniteration Analysis. An iterative scheme for the MoM-PO hybrid technique is proposed in [4] which is 
faster but in the same level of accuracy compared to the conventional MoM-PO method. The formulation is a bit different from (5) which is shown as

$$
\begin{aligned}
\mathbf{Z}_{\mathrm{MoM}} \mathbf{I}_{\mathrm{MoM}}^{i+1}= & \mathbf{V}_{\mathrm{MoM}}+\mathbf{A}_{\mathrm{MoM}-\mathrm{PO}} \mathbf{I}_{\mathrm{PO}} \\
& +\mathbf{A}_{\mathrm{MoM}-\mathrm{PO}} \mathbf{B}_{\mathrm{PO}-\mathrm{MoM}} \mathbf{I}_{\mathrm{MoM}}^{i},
\end{aligned}
$$

where $\mathbf{I}_{\mathrm{MoM}}^{i}$ is the current weights in the MoM region in the $i$ th iteration process. An iterative process will be needed to derive the solution of (7) until the currents in the MoM region become stable. The error $\xi_{i}$ in the $i$ th step is defined as

$$
\xi_{i}=\frac{\left\|\mathbf{I}_{\mathrm{MoM}}^{i}\right\|-\left\|\mathbf{I}_{\mathrm{MoM}}^{i-1}\right\|}{\left\|\mathbf{I}_{\mathrm{MoM}}^{i-1}\right\|},
$$

where the norm is defined in the 2-norm form and $\mathbf{I}_{\mathrm{MoM}}^{i}$ stands for the current coefficients in the $i$ th iteration. The threshold value of the relative residual error $\xi_{T}$ and the maximum number of iteration $N_{\text {iter }}$ are also needed to control the iteration computation.

The previous MLFMA-PO technique [12] considers the impedance matrix of the MoM by coupling the PO contributions while the iterative method updates the voltage matrix of the MoM by PO currents until it reaches stability. If only one whole octree is built, the computational complexity of the MVP processes of $Z, A$, and $B$ is nearly in the same level since the MLFMA operations are all implemented based on the whole octree. Moreover, only one solving process is needed for the conventional MLFMA-PO technique while several solving processes are needed for the iterative MLFMA-PO method. Thus, the previous noniterative method may be a better choice if only one octree exists. However, the MVP process of $Z$ based on the newly created octree structure is much faster than the other two if the extra octree for the MoM region is built. In the iterative MLFMA-PO method, several MVP processes of $A$ and $B$ are replaced by $Z$ which makes the iterative method a more efficient solver. Then, the iterative MLFMA-PO method is suggested if two octrees are built which is also demonstrated in the numerical results.

An explanation is also given in the mathematical manner. Assume that the computation time of the MVP scanning the whole octree is $\alpha$ while the value is $\beta$ for the octree of the MoM region. Apparently, $\beta$ is much smaller than the $\alpha$. The number of the solving iterations for the conventional MLFMA-PO is defined as $n_{1}$ while the average value is $n_{2}$ $\left(n_{2} \approx n_{1}\right.$ ) for the iterative method with $m$ ( $m$ is always smaller than 10) iterations between the MoM region and PO region. Thus, the computation time $\chi_{\text {mlfma-po }}$ of the conventional MLFMA-PO method is

$$
\chi_{\mathrm{mlfma}-\mathrm{po}}=3 n_{1} \alpha .
$$

Since the value of $\beta$ is negligible compared to $\alpha$, the computation time for the iterative MLFMA-PO method can be defined as

$$
\chi_{\text {iter-mlfma-po }}=m n_{2} \beta+2 m \alpha \approx m\left(n_{1} \beta+2 \alpha\right) \approx 2 m \alpha \text {. }
$$

From the above two equations, the computation time of the iterative MLFMA-PO method based on two octrees can be estimated as

$$
\chi_{\text {iter-mlfma-po }} \approx \frac{2 m}{3 n_{1}} \chi_{\text {mlfma-po }} .
$$

Since $m$ is always much smaller than $n_{1}$, the computation time of the iterative MLFMA-PO based on two octrees will be much smaller than the previous MLFMA-PO method [12] using the same hardware.

2.3. IPO Process. In the real-world problems, the structures are always complicated and nonsmooth. Thus, the currents derived by the PO method may not be accurate enough. This could make the precision of the total solution limited. In particular for the structures with strong reflections in the PO region, the conventional MLFMA-PO technique will produce totally wrong solutions in some angles. This problem can be alleviated by introducing the iterative process for the PO region (IPO) to improve the accuracy of the PO currents.

The basic MoM-IPO principle can be sought in $[16,17]$ and it is not presented here for simplicity. However, the modified IPO method proposed in this paper is a little different from the conventional IPO. Firstly, the RWG basis functions are used to expand the electric currents to maintain the continuity. Secondly, MLFMA is also adopted to decrease the computational complexity of the mutual interaction in PO region to $O(N \log (N))$. The proposed IPO process can be defined as

$$
\mathbf{I}_{\mathrm{PO}}^{i+1}=\mathbf{C}_{\mathrm{PO}} \mathbf{I}_{\mathrm{PO}}^{i}+\mathbf{I}_{\mathrm{PO}}^{0}
$$

where $\mathbf{I}_{\mathrm{PO}}^{i}$ stands for the $i$ th currents in the PO region, $\mathbf{I}_{\mathrm{PO}}^{0}$ represents the initial currents produced by the incident wave and the currents in the MoM region, and the matrix $\mathbf{C}_{\mathrm{PO}}$ is the coupling matrix in the $\mathrm{PO}$ region. The elements in the $\mathrm{C}_{\mathrm{PO}}$ can be expressed by

$\mathrm{C}_{\mathrm{PO}, i j}= \begin{cases}0, & i=j \\ \delta\left(\widehat{t}_{i}^{+}+\widehat{t}_{i}^{-}\right) \cdot \widehat{n}_{i} \times \nabla \int_{T_{j}^{ \pm}} G\left(r, r^{\prime}\right) \mathbf{f}_{j}\left(r^{\prime}\right) d \tau^{\prime}, & i \neq j,\end{cases}$

where $\hat{t}_{i}^{+}$and $\hat{t}_{i}^{-}$are the two unit vectors which are perpendicular to the $i$ th edge lying in the plane of the triangular pairs $T_{i}^{ \pm}$in the PO region. $\delta$ denotes the shadowing effects coefficient and $T_{j}^{ \pm}$is the triangular pairs of the $j$ th RWG basis function. Note that the diagonal elements of the coupling matrix are zero since the self-interactions are not considered. In this paper, the MVP process of $\mathbf{C}_{\mathrm{PO}} \mathbf{I}_{\mathrm{PO}}^{i}$ in each iteration is accelerated by MLFMA technique to maintain the computational complexity to be $O(N \log (N))$. Compared to the conventional MLFMA for the PEC objects, implementation for IPO is straightforward except for the calculation of near-field impedance matrix and lowest-level receiving patterns. The near-field impedance matrix element can be computed based on (13). However, the lowest-level 

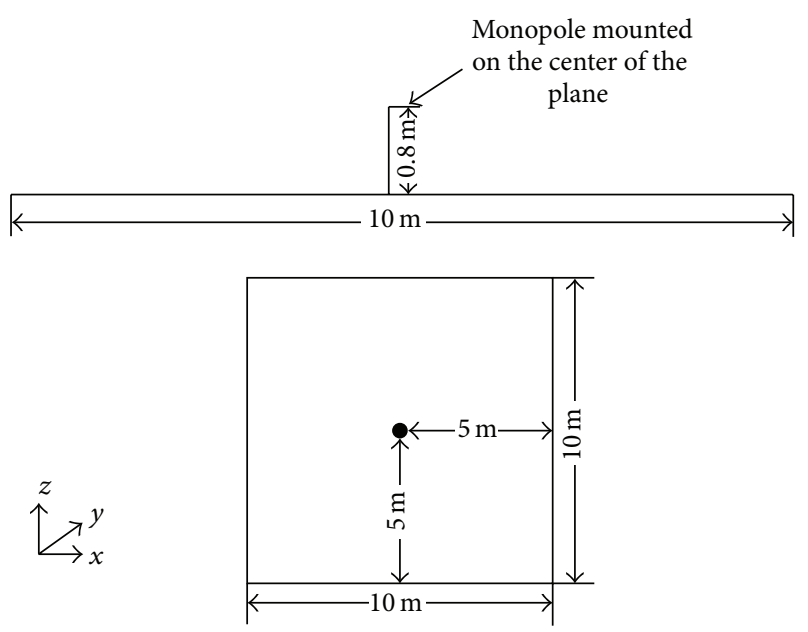

FIgURE 2: A monopole mounted on an electrically large plane.

receiving patterns need some corrections compared to the conventional MLFMA which is shown as

$$
\mathbf{R}_{\mathrm{fim}}(\widehat{k})=-\delta \widehat{k} \times\left(\widehat{t}_{i}^{+} \times \widehat{n}_{i}^{+}+\widehat{t}_{i}^{-} \times \widehat{n}_{i}^{-}\right) e^{-j \mathbf{k} \cdot \mathbf{r}_{\mathrm{im}}} .
$$

What is more, the extra octree for the PO region is not suggested to build because the PO region always plays a dominant role in the whole structure. The complexity of MVP based on the octree for the PO region will not be decreased apparently compared to the whole one.

\section{Numerical Results}

In this section, three canonical examples are examined to demonstrate the high efficiency and the reasonable accuracy of the enhanced MLFMA-PO hybrid technique compared to the previous MLFMA-PO method and conventional MLFMA. For all the experiments, the mesh sizes are about $0.1 \lambda$ ( $\lambda$ represents the wave length). The biconjugate gradient stabilized method (BiCGSTAB) equipped with the incomplete LU factorization with dual-threshold preconditioner (ILUT) is adopted as the iterative solver for the matrix equations in MoM region and the threshold of the relative residual error is chosen as 0.001 . All the computations were carried out on a workstation with a $3.4 \mathrm{GHz}$ Core i7 $4930 \mathrm{k} \mathrm{CPU}$ and $64 \mathrm{~GB}$ of RAM with OpenMP technique and the digits were stored in double precision.

3.1. A Monopole Mounted on a Large Plane. Firstly, the efficiency and accuracy of the proposed iterative technique based on two octrees without iterations in the PO region are examined. A monopole mounted on an electrically large plane is the first example, and the model is displayed in Figure 2. The work frequency of the monopole is $600 \mathrm{MHz}$. The monopole and a part of the plane which is near to the monopole are divided into the MoM region and the remaining part is given to the $\mathrm{PO}$ region. The numbers of unknowns for the MoM region and PO region are 3779 and 117110 , respectively.
TABLE 1: Calculation time and memory requirement of different methods for the monopole mounted on a plane.

\begin{tabular}{lcc}
\hline Methods & Memory (GB) & $\begin{array}{c}\text { Calc. time } \\
\text { (seconds) }\end{array}$ \\
\hline MLFMA & 6.38 & 435.2 \\
MLFMA-PO (1 tree) & 1.46 & 103.1 \\
MLFMA-PO (2 trees) & 1.50 & 82.5 \\
Iter-MLFMA-PO (1 tree) & 1.46 & 171.9 \\
Iter-MLFMA-PO (2 trees) & 1.50 & 40.7 \\
\hline
\end{tabular}

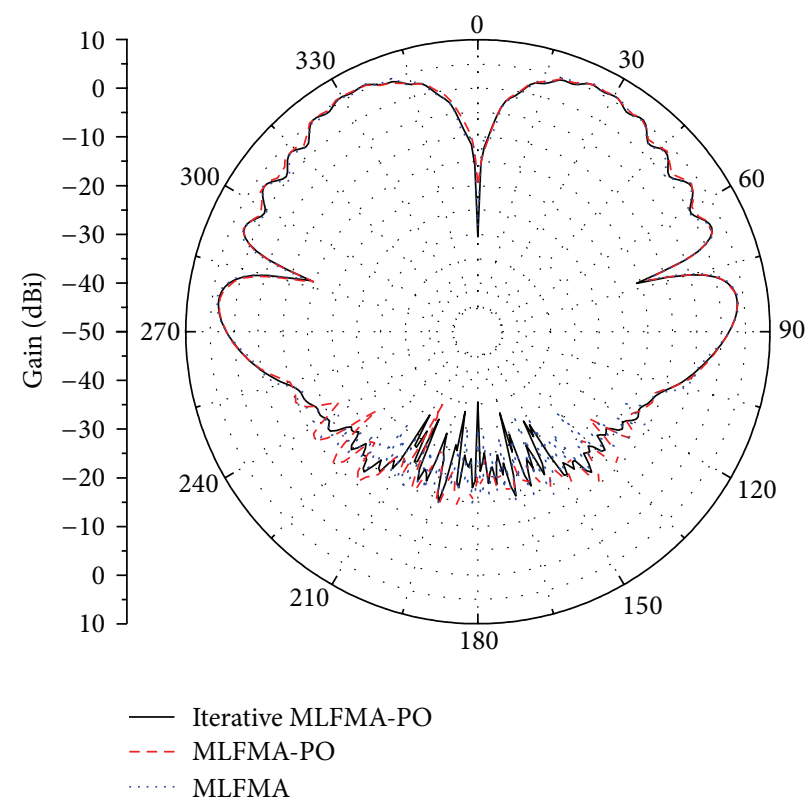

Figure 3: The far-field gain pattern in XOZ plane of the monopole mounted on the plane.

The far-field gain patterns in the XOZ cut-plane obtained from conventional MLFMA-PO technique, iterative MLFMA-PO technique, and MLFMA are shown in Figure 3. It can be seen that the three results agree well with each other. The results demonstrate that both of the MLFMA-PO techniques can achieve high accuracy compared to the lowfrequency numerical methods. Moreover, memory usage and the computation time are presented in Table 1. It is obvious that the consumed memory and calculation time of the MLFMA-PO techniques are much less than the conventional MLFMA. What is more, it is shown that the computation time can be further decreased via the extra octree built for the MoM region while the memory usage only increases from $1.46 \mathrm{~GB}$ to $1.50 \mathrm{~GB}$. The noniterative MLFMA-PO method is clearly the most efficient if only one whole octree is constructed, but the iterative MLFMA-PO method will be a better choice if the extra octree is built. Compared to the conventional MLFMA, the iterative MLFMA-PO method equipped with two octrees can achieve a speedup ratio of 10.69.

3.2. Wire Antenna Mounted on a Ship Model. Secondly, a wire antenna mounted on a ship model is studied. The ship model 

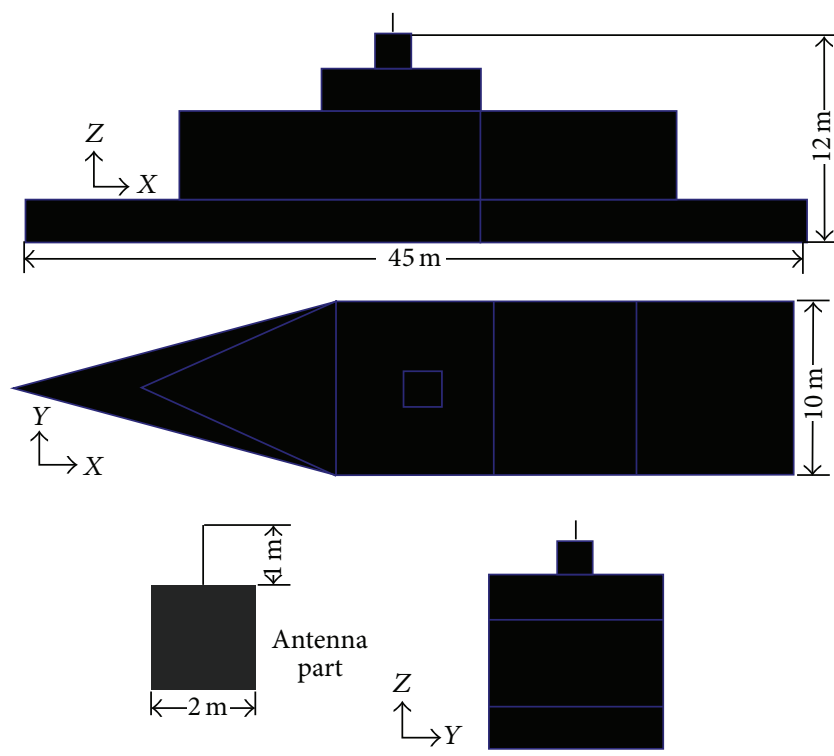

FIgURE 4: Wire antenna mounted on a ship model.

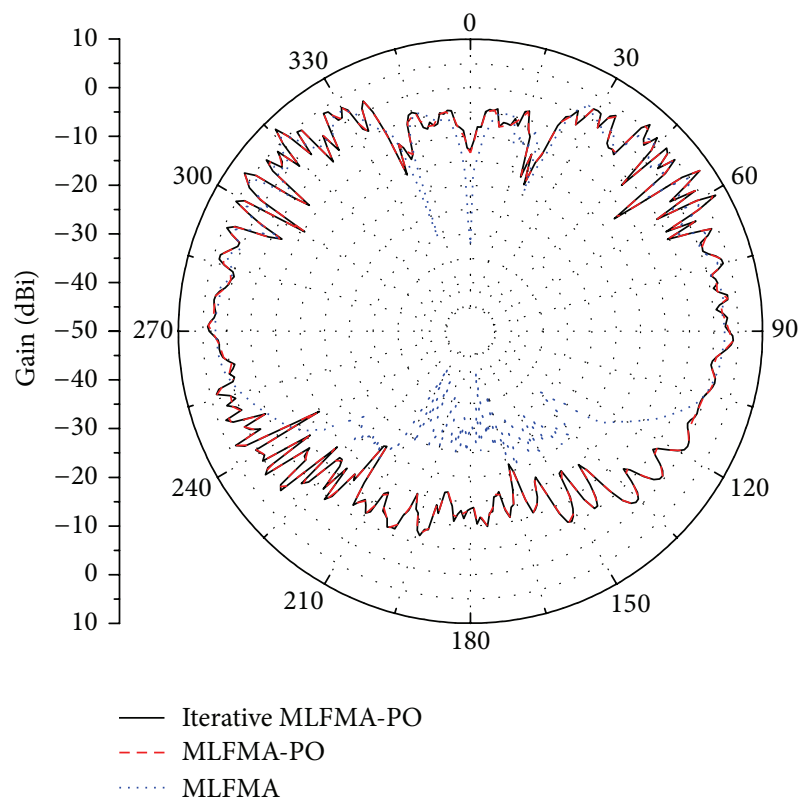

FIGURE 5: The far-field gain pattern in XOZ plane of the wire antenna mounted on ship model.

is displayed in Figure 4. The work frequency of the monopole is $300 \mathrm{MHz}$. In the hybrid analysis, the monopole, the cube, and the part of the ship near to the cube are assigned to the MoM region. The remaining part is placed into the $\mathrm{PO}$ region. The numbers of unknowns in the MoM and PO regions are 10621 and 398242, respectively.

The far-field gain patterns in the XOZ cut-plane and YOZ cut-plane obtained from conventional MLFMA-PO technique, iterative MLFMA-PO technique, and MLFMA are shown in Figures 5 and 6 . We can see that the three curves agree well with each other in the figures and the results

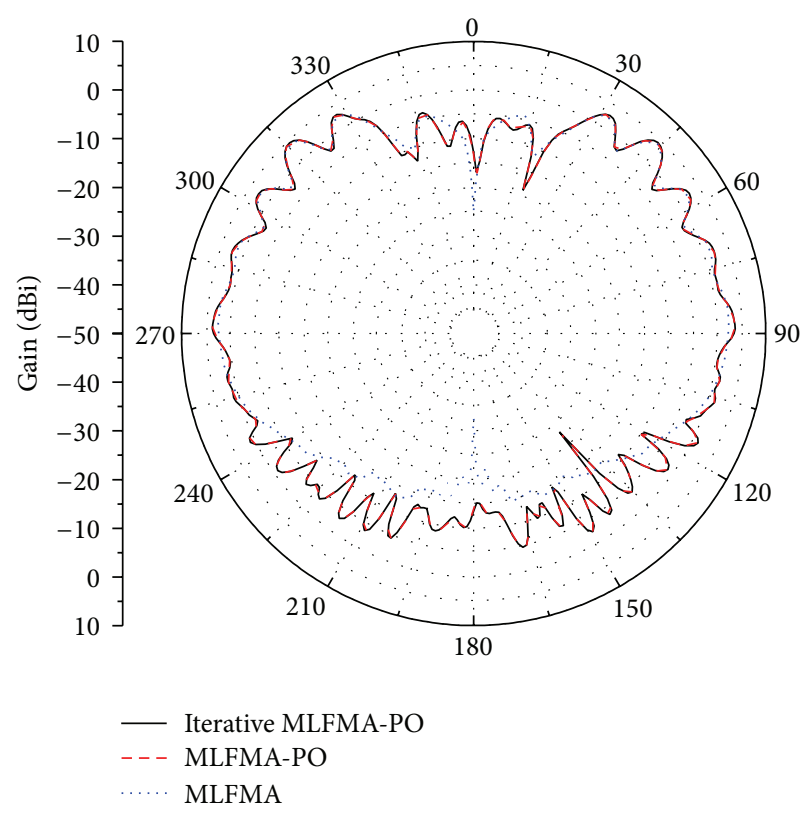

FIGURE 6: The far-field gain pattern in YOZ plane of the wire antenna mounted on ship model.

TABle 2: Calculation time and memory requirement of different methods for the monopole mounted on a plane.

\begin{tabular}{lcc}
\hline Methods & Memory (GB) & $\begin{array}{c}\text { Calc. time } \\
\text { (seconds) }\end{array}$ \\
\hline MLFMA & 35.68 & 14772.6 \\
MLFMA-PO (1 tree) & 6.25 & 1605.7 \\
MLFMA-PO (2 trees) & 6.31 & 941.4 \\
Iter-MLFMA-PO (1 tree) & 6.25 & 2376.5 \\
Iter-MLFMA-PO (2 trees) & 6.31 & 155.7 \\
\hline
\end{tabular}

derived by the two hybrid schemes are almost the same. Moreover, memory usage and the computation time are presented in Table 2. It is also demonstrated that the consumed memory and calculation time of the hybrid techniques are much less than the conventional MLFMA. The memory usage increases from $6.25 \mathrm{~GB}$ to $6.31 \mathrm{~GB}$ for the extra octree structure which can be negligible. Compared to the conventional MLFMA, the iterative MLFMA-PO method equipped with two octrees can be nearly 100 times faster than MLFMA while the speedup ratio is only 9.2 for the conventional hybrid method.

3.3. A Monopole Installed on Folding Planes. Thirdly, the accuracy of the proposed technique with IPO which is called MLFMA-IPO is studied. A monopole installed on a large twoplates body is analyzed which is shown in Figure 7. The work frequency of the monopole is also $600 \mathrm{MHz}$. The monopole and a small part of one plane which is near to the monopole are divided into the MoM region and the remaining part is given to the PO region. The numbers of unknowns for the MoM region and PO region are 7052 and 233783, respectively. 
TABLE 3: Calculation time and memory requirement of the two different methods for the monopole mounted on the folding planes.

\begin{tabular}{|c|c|c|c|c|}
\hline \multirow{2}{*}{ Methods } & \multicolumn{2}{|c|}{ Unknowns } & \multirow{2}{*}{ Memory (GB) } & \multirow{2}{*}{ Calc. time (s) } \\
\hline & MoM region & PO region & & \\
\hline MLFMA & 240835 & 1 & 18.58 & 2142.5 \\
\hline MLFMA-PO & 7052 & 233783 & 2.88 & 342.6 \\
\hline MLFMA-IPO & 7052 & 233783 & 4.29 & 141.7 \\
\hline
\end{tabular}

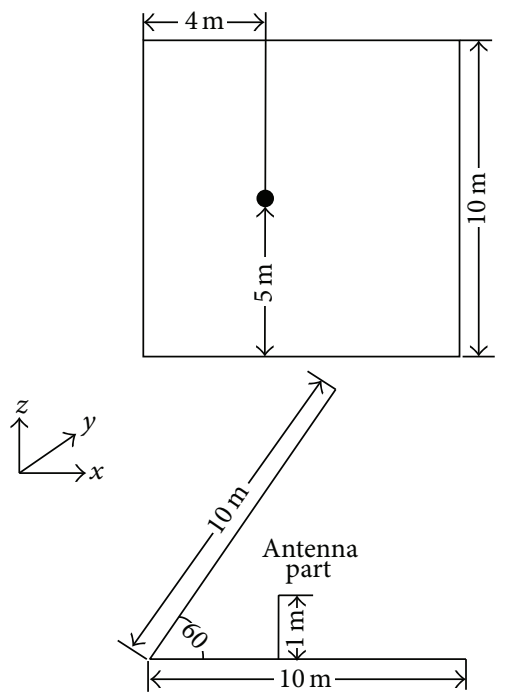

FIGURE 7: A monopole installed on folding planes.

The maximum iteration number and relative error threshold for IPO are set to be 3 and 0.1 , respectively.

In Figure 8, the far-field gain patterns in the XOZ cutplane obtained from MLFMA-IPO technique, MLFMA-PO technique, and MLFMA are plotted. It demonstrates that the results derived from the MLFMA-IPO agree well with results derived from the MLFMA while the ones derived from the conventional MLFMA-PO are not accurate in some angles (especially around 180 degrees). In Table 3 , the number of unknowns, memory usage, and the computation time are presented. It is shown that the consumed memory and calculation time of the MLFMA-IPO and MLFMA-PO are much less than the conventional MLFMA. The proposed MLFMA-IPO method uses a little more memory compared to the MLFMA-PO. However, the MLFMA-IPO method is still much faster than the conventional MLFMA-PO method owing to the extra octree structure and the iteration scheme.

\section{Conclusion}

Some modifications are developed based on the previous proposed MLFMA-PO technique to improve the accuracy and efficiency of the analysis of the electrically large objects. MLFMA is adopted to accelerate all the existing MVP processes in the newly developed hybrid technique to ensure the low computational complexity. An extra octree for the $\mathrm{MoM}$ region and alternative iterative MLFMA-PO are also implemented and analyzed. What is more, a modified IPO

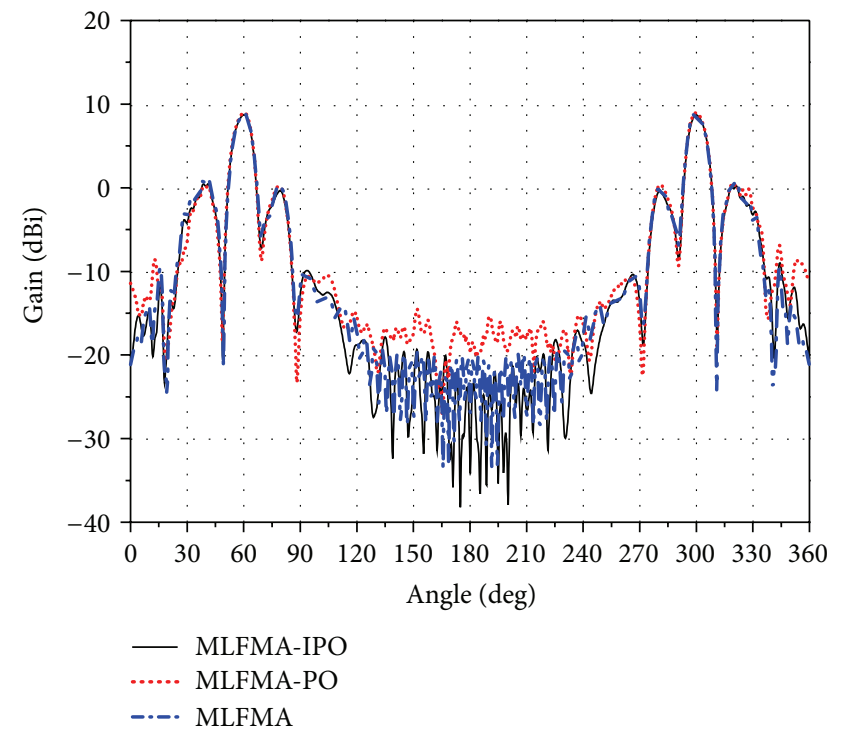

FIGURE 8: The far-field gain pattern in XOZ plane of the monopole installed on the folding planes.

method is also implemented in the PO region to improve the accuracy of the structures with strong reflections. Numerical results demonstrate the improved accuracy and efficiency of the proposed method compared to the conventional methods.

\section{Conflict of Interests}

The authors declare that there is no conflict of interests regarding the publication of this paper.

\section{Acknowledgment}

This work was supported in part by the National Natural Science Foundation of China under Grant no. 61171035.

\section{References}

[1] R. F. Harrington, Field Computation by Moment Methods, McMillan, New York, NY, USA, 1968.

[2] U. Jakobus and F. M. Landstorfer, "Improved PO-MM hybrid formulation for scattering from three-dimensional perfectly conducting bodies of arbitrary shape," IEEE Transactions on Antennas and Propagation, vol. 43, no. 2, pp. 162-169, 1995.

[3] X.-W. Zhao, Y. Zhang, H.-W. Zhang et al., "Parallel MoM-PO method with out-of-core technique for analysis of complex 
arrays on electrically large platforms," Progress in Electromagnetics Research, vol. 108, pp. 1-21, 2010.

[4] Z.-L. Liu and C.-F. Wang, "Efficient iterative method of moments-physical optics hybrid technique for electrically large objects," IEEE Transactions on Antennas and Propagation, vol. 60, no. 7, pp. 3520-3525, 2012.

[5] M. Chen, X.-W. Zhao, Y. Zhang, and C.-H. Liang, "Analysis of antenna around nurbs surface with iterative MoM-PO technique," Journal of Electromagnetic Waves and Applications, vol. 20, no. 12, pp. 1667-1680, 2006.

[6] Y. Zhang, X. W. Zhao, M. Chen, and C. H. Liang, "An efficient MPI virtual topology based parallel, iterative MoM-PO hybrid method on PC clusters," Journal of Electromagnetic Waves and Applications, vol. 20, no. 5, pp. 661-676, 2006.

[7] M. Djordjevc and B. M. Notaros, "Higher order hybrid method of moments-physical optics modeling technique for radiation and scattering from large perfectly conducting surfaces," IEEE Transactions on Antennas and Propagation, vol. 53, no. 2, pp. 800-813, 2005.

[8] J. Song, "Multilevel fast multipole algorithm for electromagnetic scattering by large complex objects," IEEE Transactions on Antennas and Propagation, vol. 45, no. 10, pp. 1488-1493, 1997.

[9] J. R. Phillips and J. K. White, "A precorrected-FFT method for electrostatic analysis of complicated 3-d structures," IEEE Transactions on Computer-Aided Design of Integrated Circuits and Systems, vol. 16, no. 10, pp. 1059-1072, 1997.

[10] X. Wang, S.-X. Gong, J. Ma, and C.-F. Wang, "Efficient analysis of antennas mounted on large-scale complex platforms using hybrid AIM-PO technique," IEEE Transactions on Antennas and Propagation, vol. 62, no. 3, pp. 1517-1523, 2014.

[11] L. Gurel, A. Manyas, and O. Ergul, "PO-MLFMA hybrid technique for the solution of electromagnetic scattering problems involving complex targets," in Proceedings of the 2nd European Conference on Antennas and Propagation, pp. 1-5, 2007.

[12] Y. Zhang and H. Lin, "MLFMA-PO hybrid technique for efficient analysis of electrically large structures," IEEE Antennas and Wireless Propagation Letters, vol. 13, pp. 1676-1679, 2015.

[13] A. I. Papadopoulos and D. P. Chrissoulidis, "A corrected physical-optics solution to 3-D wedge diffraction," Electromagnetics, vol. 20, no. 2, pp. 79-98, 2000.

[14] A. Hujanen and J. C.-E. Sten, "Study of scattering by a perfectly conducting wedge with finite sized faces," Annales des Telecommunications, vol. 56, no. 9-10, pp. 587-594, 2001.

[15] Y. Z. Umul, "Wedge diffraction in terms of the method of physical optics," IEICE Electronics Express, vol. 6, no. 11, pp. 763768, 2009.

[16] H. Q. Zhai and C. H. Liang, "A simple iterative method for considering multibounce in PO region of MoM-PO," Microwave and Optical Technology Letters, vol. 40, no. 2, pp. 110-112, 2004.

[17] J. M. Taboada and F. Obelleiro, "Including multibounce effects in the moment-method physical-optics (MMPO) method," Microwave and Optical Technology Letters, vol. 32, no. 6, pp. 435-439, 2002.

[18] S. M. Rao, D. R. Wilton, and A. W. Glisson, "Electromagnetic scattering by surfaces of arbitrary shape," IEEE Transactions on Antennas and Propagation, vol. 30, no. 3, pp. 409-418, 1982. 

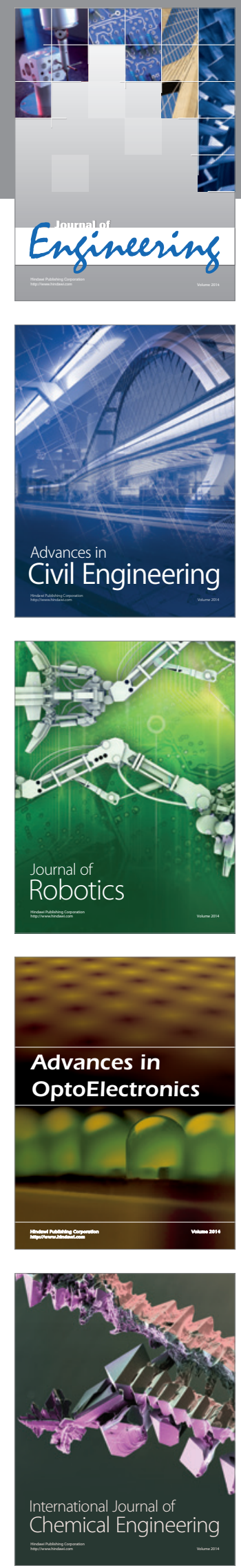

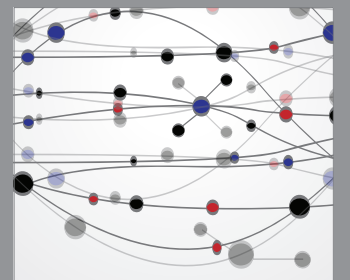

The Scientific World Journal
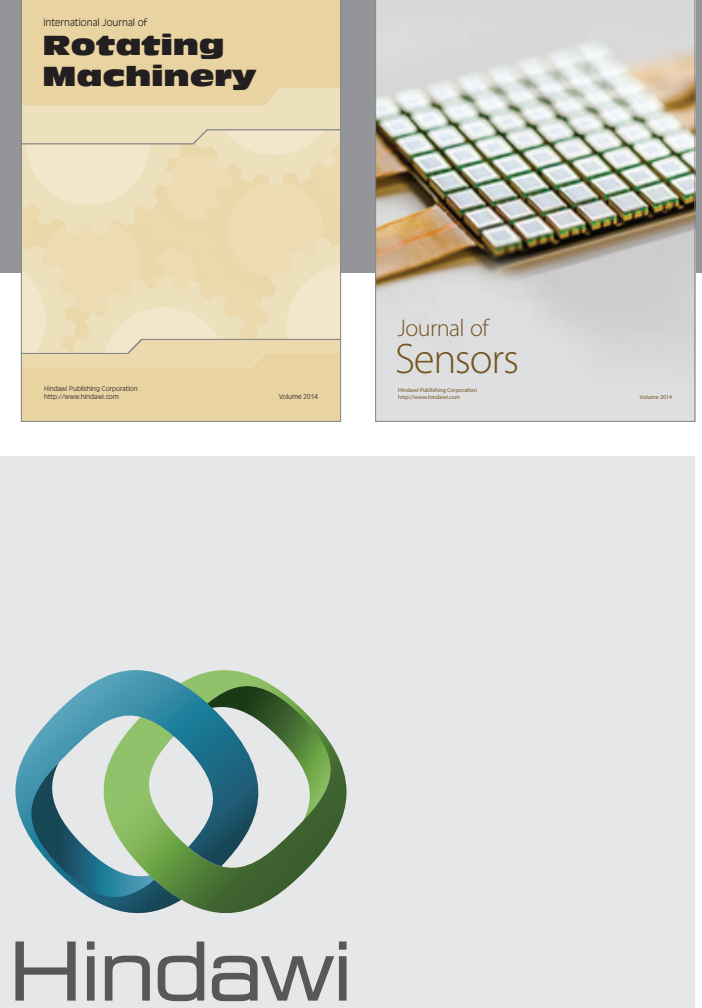

Submit your manuscripts at http://www.hindawi.com
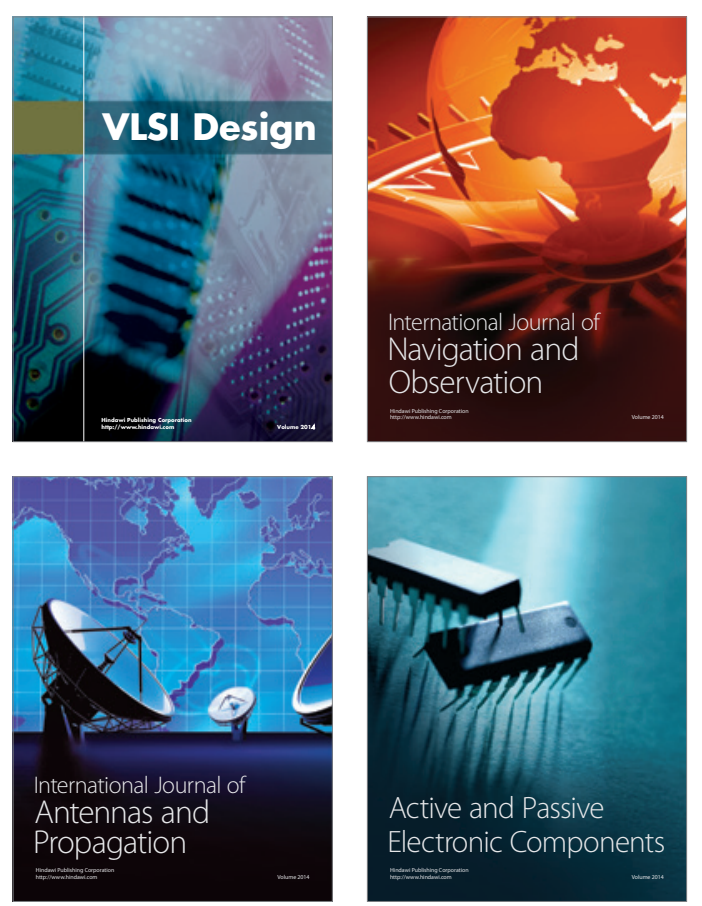
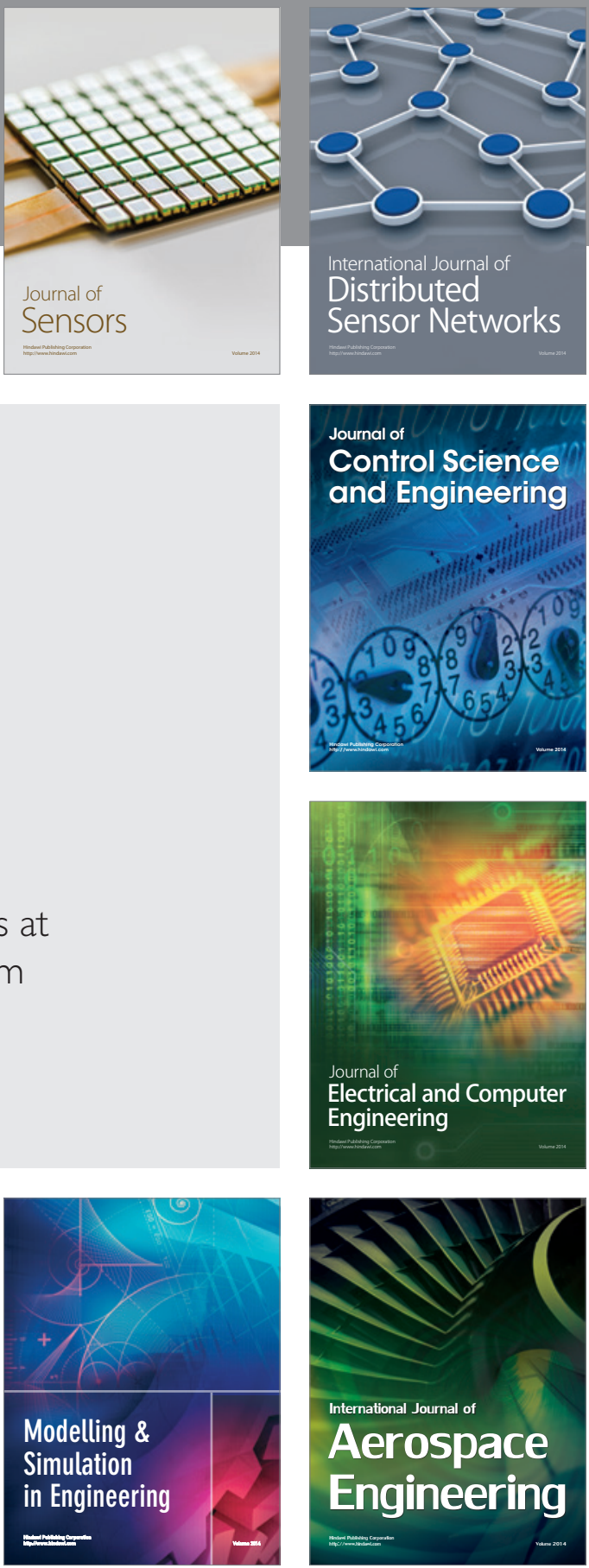

Journal of

Control Science

and Engineering
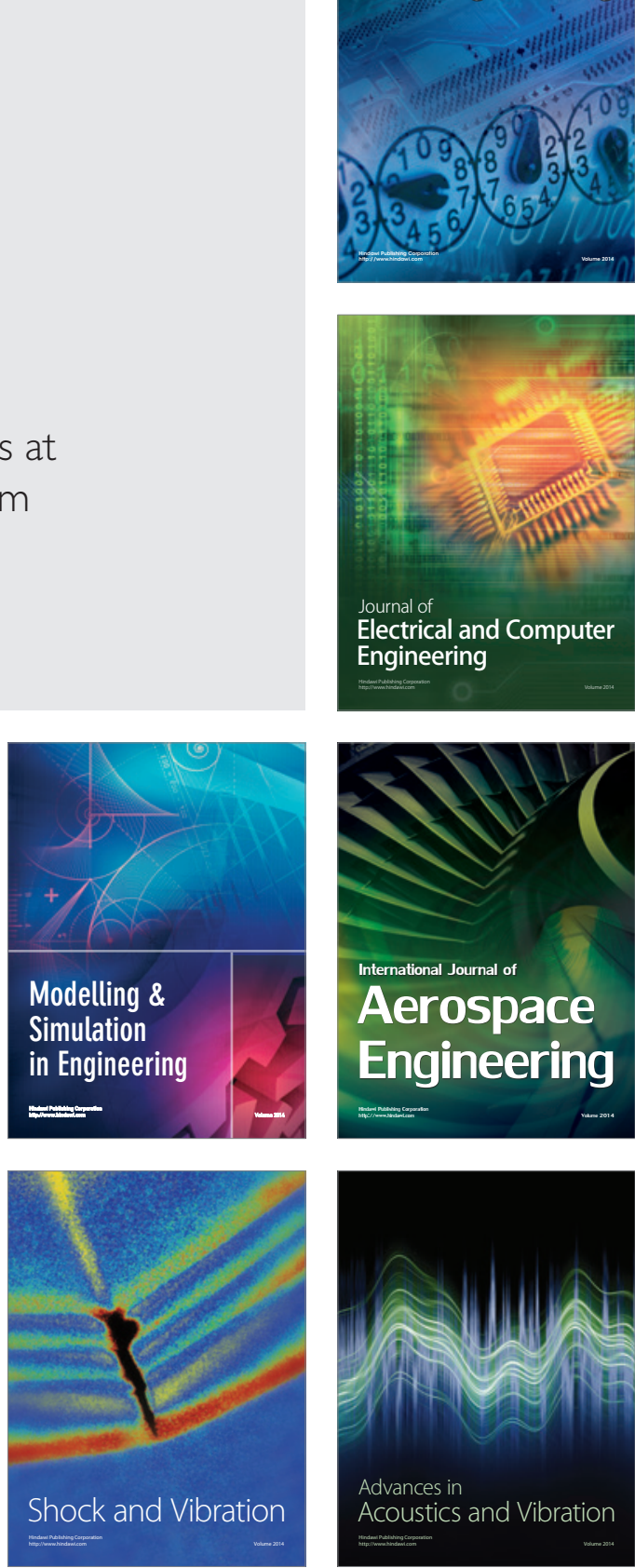